\title{
The combination therapy with EpCAM/CD3 BsAb and MUC-1/CD3 BsAb elicited antitumor immunity by T-cell adoptive immunotherapy in lung cancer
}

\author{
Ce Wang, Shang Chen, Yingjuan Wu, Di Wu, Jingbo Wang, Furong Li ${ }^{\bowtie}$ \\ Shenzhen key laboratory of stem cell research and clinical transformation, Guangdong Engineering Technology Research Center of Stem cell and Cell therapy, \\ Translational Medicine Collaborative Innovation Center, The Second Clinical Medical College (Shenzhen People's Hospital), Jinan University, Shenzhen 518020, \\ China. \\ $\square$ Corresponding author: Fu-Rong Li, Translational Medicine Collaborative Innovation Center, The Second Clinical Medical College (Shenzhen People's \\ Hospital), Jinan University, 1017 Dongmen North Road, Shenzhen 518020, China. E-mail: frli62@163.com. \\ (C) The author(s). This is an open access article distributed under the terms of the Creative Commons Attribution License (https://creativecommons.org/licenses/by/4.0/). \\ See http:/ /ivyspring.com/terms for full terms and conditions.
}

Received: 2021.04.15; Accepted: 2021.07.22; Published: 2021.07.31

\begin{abstract}
Lung cancer remains a global challenge due to high morbidity and mortality rates and poor response to treatment, and there are still no effective strategies to solve it. The bispecific antibody (BsAb) is a novel antibody, which can target two different antigens and mediate specific killing effects by selectively redirecting effector cells to the target cells. In this study, we combined two BsAbs to achieve a dual-target therapy strategy of EpCAM+ and $M U C-1^{+}$with high affinity and specificity. The results showed that the combination of two BsAbs against EpCAM and MUC-1 could inhibit the growth of lung cancer more effectively in cell lines and primary tumors. The superior antitumor effect of two BsAbs could be attributable to enhanced CTL and increased production of type I IFNs. At the same time, the combination of EpCAM/CD3 BsAb and MUC-1/CD3 BsAb significantly regulated $T$ population in the TDLNs. Therefore, we have found a potential immunotherapeutic strategy, which was the combination therapy with EpCAM/CD3 BsAb and MUC-1/CD3 BsAb for the treatment of non-small cell lung cancer.
\end{abstract}

Key words: non-small cell lung cancer; EpCAM/CD3 BsAb; MUC-1/CD3 BsAb; immunotherapy

\section{Introduction}

Lung cancer was one of the most common malignant tumors in the world [1], whose morbidity and mortality were the highest among all the malignant tumors. Of all the lung cancer patients, $80 \%-85 \%$ suffered from non-small cell lung cancer (NSCLC). In recent years, the incidence of NSCLC was younger and rising worldwide. Although the clinical diagnosis and treatment level was developing rapidly, the 5-year survival rate of NSCLC is only $15 \%$ [2-3]. Therefore, there was an urgent need to study the feasibility of anti-lung cancer treatment.

Because BsAbs could target two different antigens, they were widely used in the field of tumor therapy. In the past decade, the research of BsAb has been growing steadily. Immune cells played an important role in the immunotherapy of tumor. Mature T cells marked with CD3 played an important role in the immune response and became the preferred target cells for the study of effect of BsAb [4]. At present, catumaxomab, which was approved by EU in 2009, was the first BsAb targeted at EpCAM/CD3 for cancer treatment [5]. The anti-tumor effect of catumaxomab was achieved by recruiting $\mathrm{T}$ cells to kill target cells, using antibody dependent cell-mediated cytotoxicity and cytokine-mediated cytotoxicity [6]. In addition, the clinical trials for ovarian cancer, gastric cancer, non-small cell lung cancer and breast cancer were also in progress [7]. Another commercially available monoclonal antibody was blinatumomab, which could specifically bind $\mathrm{CD}^{+}{ }^{+} \mathrm{T}$ lymphocytes and $\mathrm{CD} 19^{+} \mathrm{B}$ lymphocytes to mediate high level cytotoxic effect of $\mathrm{T}$ cells on lymphoma cells [8]. The results showed that BsAbs have obvious advantages. They had play an effective killing effect at very low concentration of antibody drugs. However, tumors were usually pathological 
changes caused by various reasons, regulated by multiple signal pathways, and having more than one surface specific antigen. As a result, blocking multiple targets at the same time might achieve better therapeutic effect [9]. Therefore, we combined two BsAbs for anti-tumor treatment. In addition, it was still a problem to be solved to determine the best time and treatment course for the use of antibody drugs.

As we all know, epithelial cell adhesion molecule (EpCAM) was a single transmembrane protein of adhesion molecule family. It was highly expressed in the basement membrane of many kinds of malignant tumors, and abnormally expressed in many kinds of human tumor tissues such as colorectal cancer, prostate cancer and gastric cancer. It participated in the process of proliferation, differentiation, adhesion, invasion and migration of tumor cells by regulating related signal pathways or target genes. It was closely related to the occurrence and development of tumors [10-12]. It has been reported that high expression of EpCAM was closely related to lung cancer metastasis and poor prognosis, thus might play an important role in the occurrence and development of lung cancer [13]. In addition, Pak et al. found that the positive expression rate of EpCAM protein in tumor tissues of NSCLC patients in stage II-IV was $73.33 \%$, significantly higher than $47.06 \%$ in stage I of NSCLC patients [14].

In addition, polymorphic epithelial mucin (MUC-1) was a kind of high molecular weight glycoprotein, which had abnormal expression in epithelial cell-derived malignant tumors and played an important role in tumor growth, invasion, development and metastasis [15]. Giatromanelaki et al. found that in NSCLC, the expression of MUC-1 was an important prognostic factor independent of $\mathrm{T}$ and $\mathrm{N}$ stages, which was related to the expression of vascular endothelial growth factor (VEGF), which leads to angiogenesis and tumor migration [16]. The expression of MUC-1 in lung cancer could provide effective information for clinical judgment of lymph node metastasis trend, prediction of lymph node metastasis potential and judgment of the prognosis of NSCLC, and played a guiding role in prognosis and postoperative treatment of NSCLC [17].

In this study, we combined EpCAM/CD3 BsAb and MUC-1/CD3 BsAb to target both EpCAM and MUC-1 on the surface of tumor cells. The results showed that the combination of two BsAbs could effectively inhibit the growth of NSCLC and promote the apoptosis of tumor cells. We further confirmed that the combination of BsAbs therapy could effectively inhibit tumor growth and inflammatory response in vivo, suggesting its immunotherapeutic potential in solid tumor therapy in vivo.

\section{Methods}

\section{Materials}

Human blood buffy coats from healthy donors were obtained from Shenzhen Blood Center (Shenzhen, China), and tested negative for blood-born pathogens using the standard protocols of the blood center. All of the protocols involving human blood samples were approved by the Institutional Review Board of the Shenzhen Blood Center. 5-6 weeks old female NOD/SCID mice were purchased from Gem Pharmatech LLC (Jiangsu, China), 5-6 weeks old female BALB/c mice were purchased from Guangdong Province Laboratory Animal Center (Guangzhou, China) and maintained in the institutional animal care facility. All animal protocols were approved by Institutional Animal Care and Usage Committee of Shenzhen People's Hospital.

\section{Sample Collection}

5 patients with primary lung cancer from January 2016 to June 2019 in Shenzhen People's Hospital. Informed consent for the additional core-needle biopsy and experimental use of tumor samples was obtained from all patients, following a protocol approved by the Ethics Committee of Shenzhen People's Hospital.

\section{The EpCAM/CD3 BsAb and the MUC-1/CD3 BsAb}

The EpCAM/CD3 BsAb and the MUC-1/CD3 $\mathrm{BsAb}$ in this study were obtained from BenHealth Biopharmaceutical (Shenzhen) Co. LTD. The EpCAM/CD3 BsAb or the MUC-1/CD3 BsAb was connected MUC1 antibody or EpCAM antibody and CD3 antibody by biodegradable nanomaterials. Briefly, MUC1/CD3 BsAb or EpCAM/CD3 BsAb were obtained by using 1-Ethyl-3-(3-dimethylaminopropyl) carbodiimide (EDC. $\mathrm{HCl}$ ) and N-hydroxysuccinimide (NHS) to bind MUC1 antibody or EpCAM antibody and CD3 antibody to polylactic acid glycolic acid (PLGA), respectively.

\section{Cell lines and cell culture conditions}

A549, H466 and H1975 cells were obtained from Shanghai Institutes for Biological Sciences Cell Bank (Shanghai, China), and maintained in Dulbecco's Modified Eagle Medium (DMEM high glucose, Invitrogen, CA, USA) supplemented with $10 \%$ fetal bovine serum (FBS, Thermo Scientific, MA, USA). All cells were incubated at a $37{ }^{\circ} \mathrm{C}$ humidified incubator containing $5 \% \mathrm{CO}_{2}$.

\section{T cells culture, stimulation and activation}

Human monocytes were enriched by plastic adherence of peripheral blood mononuclear cells 
(PBMCs) in a $100 \mathrm{~mm}$ dish at $37^{\circ} \mathrm{C}, 5 \% \mathrm{CO}_{2}$. After $2 \mathrm{~h}$ of incubation, the nonadherent cells were removed. The activated $\mathrm{T}$ cells (aT cells) were harvested after cultured with anti-human CD3 antibody $(1 \mu \mathrm{g} / \mathrm{ml})$ and anti-human CD28 antibody $(1 \mu \mathrm{g} / \mathrm{ml})$ followed by IL-2 $(100 \mathrm{IU} / \mathrm{ml})$ for $72 \mathrm{~h}$. And then the aT cells were treated with or without EpCAM/CD3 BsAb (10 $\mu \mathrm{g} / \mathrm{ml})$ or MUC-1/CD3 BsAb $(10 \mu \mathrm{g} / \mathrm{ml})$ followed by IL-2 $(100 \mathrm{IU} / \mathrm{ml})$ for $72 \mathrm{~h}$, and then washed twice by PBS to acquire different BsAb treated aT cells (Med: medium, EpCAM: EpCAM/CD3 BsAb, MUC-1: MUC-1/CD3 BsAb and EpCAM \& MUC-1: EpCAM/ CD3 BsAb \& MUC-1/CD3 BsAb).

\section{The binging efficiency of $T$ cells and tumor cells}

A549, H466 and H1975 cells were labeled with Dil $(2 \mu \mathrm{g} / \mathrm{ml})$. The different BsAb treated T cells were labeled with Calcein-AM $(2 \mu \mathrm{M})$. The activated T cells and target cells (A549, H466 and H1975) were cultured in 24 well plates with effect/target (E: T) ratio of 10: 1 for $6 \mathrm{~h}$, and then the cells were harvested. The binging efficiency of cells was detected by flow cytometry.

\section{Cytokines ELISA assay}

A549, H466 and H1975 cells or primary tumor cells were exposed to different BsAb treated T cells for $72 \mathrm{~h}$. The supernatant of the culture medium was collected. The levels of IL- 6 and IFN- $\gamma$ in the medium were determined using ELISA kit (eBioscience, San Diego, CA) according to the manufacturer's instructions.

\section{Cytotoxic T-lymphocyte (CTL) response}

The different BsAb treated T cells and target cells (A549, H466 and H1975 cells) were cultured in 96-well plates at various effector/target (E: T) ratios for $6 \mathrm{~h}$. The LDH activity in supernatants was quantified using Cytotox96 Non-Radioactive Cytotoxicity Assay Kit (Promega, WI, USA) according to manufacturer's instruction.

Another part of H1975 cells were labeled with Dil $(2 \mu \mathrm{g} / \mathrm{ml})$. The different BsAb treated T cells were labeled with Calcein-AM $(2 \mu \mathrm{M})$. T cells and target cells (H1975) were cultured in 24 well plates with effect/target (E: T) ratio of 10:1 for $24 \mathrm{~h}$. CTL response was dynamically monitored by living cell workstation.

\section{Tumor implantation and animal immunization}

5-6 weeks old female NOD/SCID mice were subcutaneously (s.c.) injected with A549, H466 or H1975 cells $\left(5 \times 10^{6}\right.$ cells/ mouse) on right buttock. On day 7 after tumor cells implantation, mice were i.v. injected with PBS, activated T cells, or different BsAb treated activated $\mathrm{T}$ cells once a week for 3 weeks. Tumor diameters were measured in two dimensions every three days using a caliper, and the tumor volume was calculated according to the following formula: volume $\left(\mathrm{mm}^{3}\right)=(\text { width })^{2} \times($ length $) \times 1 / 2$.

\section{Statistical analysis}

Data are reported as the mean SE. The differences between the control and experimental groups were assessed using a Student's t test, and the differences among the multiple groups were analyzed using one-way ANONA (Graphpad Prism, GraphPad Software, La Jolla, CA). A value of $\mathrm{p}<0.05$ was considered statistically significant.

\section{Results and Discussion}

\section{The expression of EpCAM and MUC-1 by tumor cells}

First, we evaluated EpCAM and MUC-1 expression in five human lung cancer cell lines using flow cytometry. All cell lines expressed MUC-1 at relative high levels (Fig. 1), but only H466 (Fig. 1E) and H1975 (Fig. 1F) expressed EpCAM at relative high levels. Hence, H466 cells and H1975 cells were selected as experimental groups in this study, both expressed relative high levels of EpCAM and MUC-1, and A549 cells were selected as control group.

\section{The effects of EpCAM/CD3 BsAb and MUC-1/ CD3 BsAb on the binding efficiency of $T$ cells and tumor cells}

Our results showed that the binding efficiency of treated T cells by EpCAM/CD3 BsAb alone to A549, $\mathrm{H} 466$ or $\mathrm{H} 1975$ was $7.8 \%, 41.9 \%$ and $60.3 \%$ respectively, and the binding efficiency of treated $\mathrm{T}$ cells by MUC-1/CD3 BsAb alone to A549, H466 or $\mathrm{H} 1975$ was $62.0 \%, 52.2 \%$ and $57.6 \%$ respectively. Remarkably, the binding efficiency was positively correlated with of the expression of EpCAM or MUC-1 in tumor cells (Fig. 2D-F).

\section{The anti-cancer effect of combination of EpCAM/CD3 BsAb and MUC-1/CD3 BsAb in lung cell lines}

We have combined two kinds of BsAbs targeting both EpCAM and MUC-1 on the surface of lung tumors cell lines. Herein, the anti-cancer effects of the combination of EpCAM/CD3 BsAb and MUC-1/CD3 $\mathrm{Bs} \mathrm{Ab}$ were evaluated in vitro. When target/effect ratio to 10:1, EpCAM/CD3 BsAb alone or MUC-1/ CD3 BsAb alone significantly enhance CTL activity in H466 cells and H1975 cells, which was similar to target/effect ratio to 5:1 (Fig. 3B-C). Notably, combination of EpCAM/CD3 BsAb and MUC-1/CD3 $\mathrm{BsAb}$ appeared to be more potent than that by 
EpCAM/CD3 BsAb or MUC-1/CD3 BsAb alone to CTL activity (Fig. 3B-C). More interestingly, EpCAM/CD3 BsAb alone could not effectively enhance CTL activity, however, MUC-1/CD3 BsAb alone or combination of EpCAM/CD3 BsAb and MUC-1/CD3 BsAb could enhance CTL activity (Fig. 3A) in A549. The results might be due to the fact that the expressions of EpCAM at low level in A549 (Fig. 1), previous study confirmed that some non-small cell lung cancer (NSCLC) tumor cells had a lower expression of EpCAM [18]. Therefore, it was difficult to effectively antitumor therapy with EpCAM/CD3 $\mathrm{BsAb}$ for EpCAM low expression of tumor. These results further suggested more effective CTL has been activated by using the combination of EpCAM/CD3 $\mathrm{BsAb}$ and MUC-1/CD3 BsAb than EpCAM/CD3 BsAb or MUC-1/CD3 BsAb alone.

In the present study, we dynamically observed anti-cancer effect of combination of EpCAM/CD3 BsAb and MUC-1/CD3 BsAb in H1975 through the living cell workstation. H1975 were labeled with Dil as red fluorescence, while different BsAb treated $\mathrm{T}$ cells were labeled with Calcein-AM as green fluorescence. The results showed that the green ${ }^{+} \mathrm{T}$ cells directionally moved towards the red ${ }^{+} \mathrm{H} 1975$ and continued to adsorb around the red ${ }^{+}$H1975 until the red fluorescence gradually disappeared
(Supplementary Video S1), confirming CTL response induced by combination of EpCAM/CD3 BsAb and MUC-1/CD3 BsAb. Hence, as a result difference of surface molecular of different cancer cell lines (Fig. 1), the combination of two BsAbs could increase the killing effect of tumor, thereby contributing to anti-cancer therapy.

To further confirm the anti-tumor effect of BsAb, we detected the CTL response from $1 \mathrm{~h}$ to $6 \mathrm{~h}$. The results showed that the CTL response increased significantly with the increased treatment time of BsAb. It was worth noting that the BsAb has produced a significant CTL response at 2 hours. Therefore, tumor specific CTL response played an important role in the anti-tumor effect of the combination therapy with EpCAM/CD3 BsAb and MUC-1/CD3 BsAb.

\section{Proinflammatory effects of combination of EpCAM/CD3 BsAb and MUC-1/CD3 BsAb in lung cell lines}

The effects of combination of EpCAM/CD3 $\mathrm{BsAb}$ and MUC-1/CD3 BsAb on cytokine production were further confirmed using ELISA in lung cell lines. As shown in Fig. 4, both EpCAM/CD3 BsAb alone and MUC-1/CD3 BsAb alone markedly increased the production of IFN- $\gamma$ (Fig. 4B-C) and IL-6 (Fig. 4E-F),
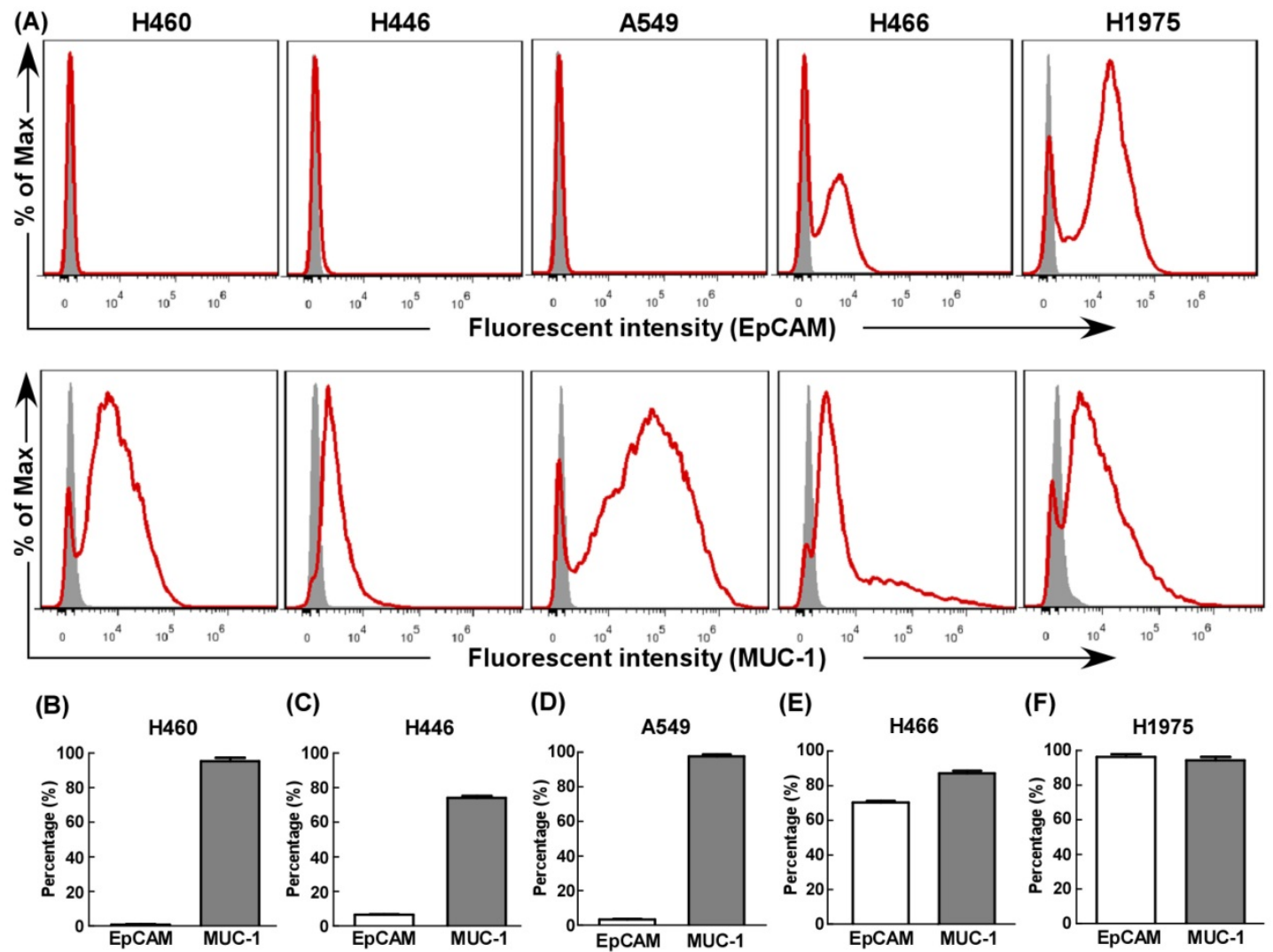

(C) $\quad$ (D)
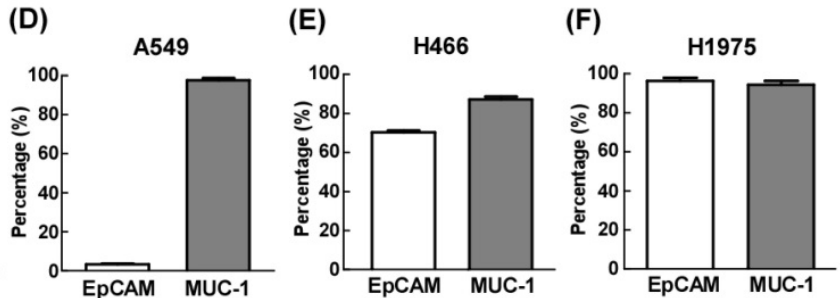

Figure 1. The expression of EPCAM and MUC-1 in lung tumor cell lines. The expression of EpCAM and MUC-1 were measured using flow cytometry in H460 (B), H446 (C), A549 (D), H466 (E) and H1975 (F). Experiments were repeated three times in triplicate each time $(n=3)$. 
suggesting that there was no significant difference between the two treatments in H466 and H1975. However, when the target/effect ratio to $5: 1$ or 10:1, MUC-1/CD3 BsAb alone markedly increased the production of IFN- $\gamma$ (Fig. 4A) and IL-6 (Fig. 4D), EpCAM/CD3 BsAb alone hardly up-regulated IFN-ץ (Fig. 4A) and IL-6 (Fig. 4D), which might be a result of the low expression of EpCAM in A549 cells. Notably, combination of EpCAM/CD3 BsAb and MUC-1/CD3 $\mathrm{BsAb}$ appeared to be more potent than that by EpCAM/CD3 BsAb or MUC-1/CD3 BsAb alone to elevate the production of IFN-ץ (Fig. 4A-C) and IL-6 (Fig. 4D-F) in A549, H466 and H1975. Overall, combination of EpCAM/CD3 BsAb and MUC-1/CD3 $\mathrm{BsAb}$ induced stronger cytokine production in A549, H466 and H1975. Besides, the proinflammatory effects of BsAbs on cancer cells appeared to be associated with the expression of specific antibodies on the surface of cancer cells, and there was a dose dependence.
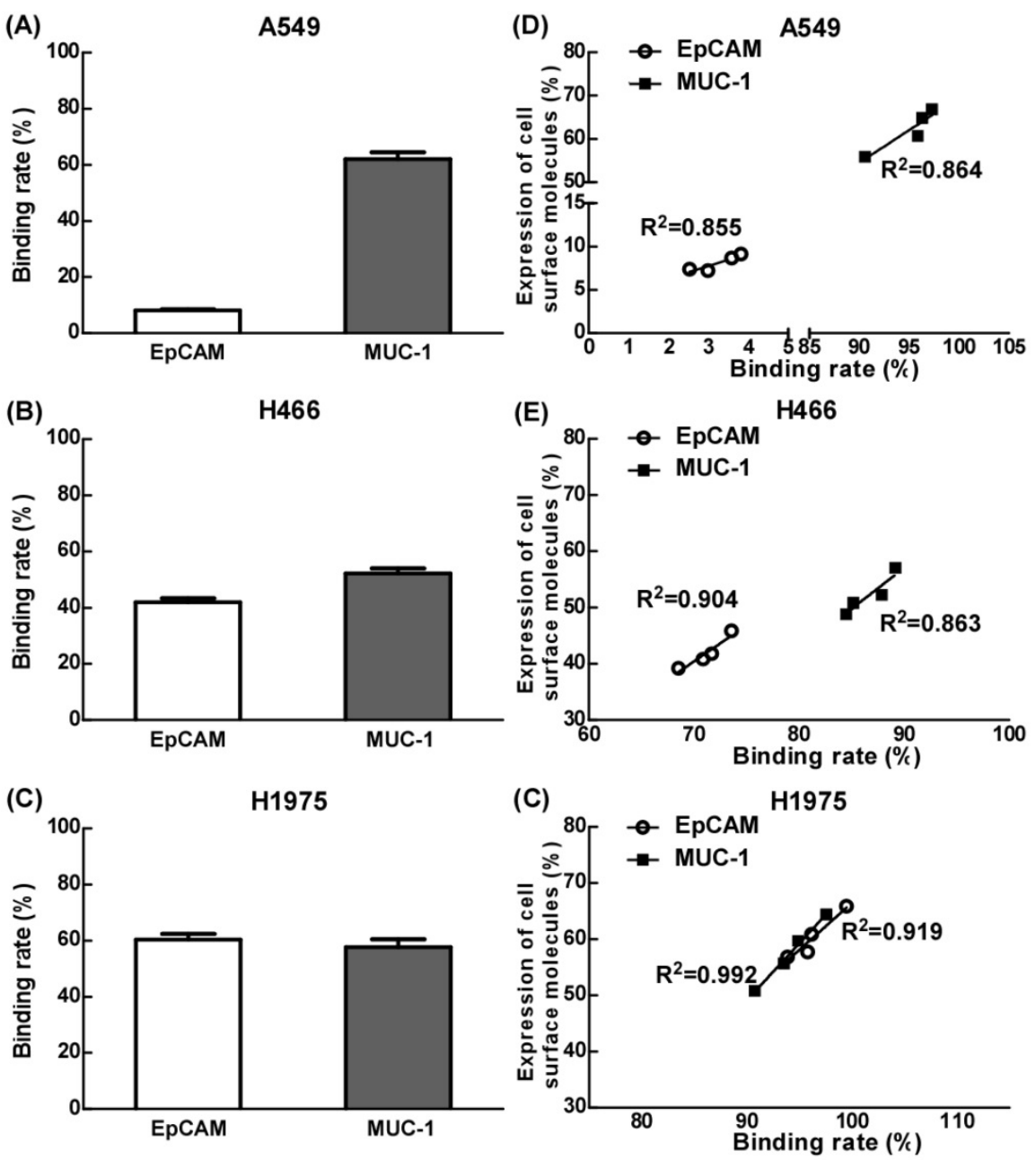

Figure 2. The effect of EpCAM/CD3 BsAb complexed with MUC-1/CD3 BsAb on the binding efficiency of $T$ cells and tumor cells. A549, H466 and H1975 cells were labeled with Dil $(2 \mu \mathrm{g} / \mathrm{ml})$. The different BsAbs treated T cells were labeled with Calcein-AM $(2 \mu \mathrm{M})$. The binding rate of T cells (green) and tumor cells (red) by double positive cells was recorded using quantified using flowcytometry $(A-C)$. The correlation between binding rate $(X$-axis) and expression of cell surface molecules rate (Y-axis) in A549 (D), H466 (E) and H1975 (F). Bars shown are mean \pm SE ( $n=3-4)$.

\section{The expression of EpCAM and MUC-1 by primary tumor cells}

Further, we evaluated EpCAM and MUC-1 expression by primary tumor cells using flow cytometry. Our results showed that the primary tumor cells expressed MUC-1 and EpCAM at relatively high levels, but there were significant differences among different primary tumors, the expression rates of MUC- 1 and EpCAM were $65 \%-89 \%$ and $75 \%-97 \%$ respectively (Fig. 5).

\section{The anti-cancer effect of combination of EpCAM/CD3 BsAb and MUC-1/CD3 BsAb in primary tumor cells}

Then we investigated the anti-cancer effect of the combination of EpCAM/CD3 BsAb and MUC-1/CD3 $\mathrm{BsAb}$ in primary lung tumors cells. As shown in Fig. 5, when target/effect ratio was 10:1, both EpCAM/CD3 BsAb alone and MUC-1/CD3 BsAb alone significantly enhance CTL activity in primary lung tumors cells, combination of EpCAM/CD3 BsAb and MUC-1/CD3 BsAb appeared to be more potent to CTL activity. Similarly, when target/effect ratio was 5:1, combination of EpCAM/CD3 BsAb and MUC-1/CD3 BsAb appeared to be more potent to CTL activity in primary lung tumors cells (Fig. 6). These results confirmed that the combination therapy of EpCAM/CD3 BsAb and MUC-1/CD3 BsAb has more effectively induced anti-tumor CTL response in primary tumor cells. In the present study, due to different expression levels of surface molecular in primary tumor cells (Fig. 5), combination of EpCAM/CD3 BsAb and MUC-1/CD3 BsAb could increase the killing effect of tumor, thereby contributing to anti-cancer therapy.

\section{Proinflammatory effects of combination of EpCAM/CD3 BsAb and MUC-1/CD3 BsAb in primary tumor cells}

Next, the effects of combination of EpCAM/CD3 $\mathrm{BsAb}$ and MUC-1/CD3 BsAb on cytokine production were further confirmed using ELISA 
in primary tumor cells. Our results showed that combination of EpCAM/CD3 BsAb and MUC-1/CD3 $\mathrm{BsAb}$ significantly elevated the production of IFN- $\gamma$ by $4-5$ folds than untreated $T$ cells in primary tumor (Fig. 7), which was a key cytokine indispensable to anti-tumor therapy and enhanced tumor-specific CTL responses, consistent with our previous observation [19]. In addition, lack of IFN- $\gamma$ production by $\mathrm{CD}^{+} \mathrm{T}$ cells suggested that the $\mathrm{CD}^{+} \mathrm{T}$ cell-mediated CTL response could be limited [20]. It was noteworthy that combination of EpCAM/CD3 BsAb and MUC-1/CD3 $\mathrm{BsAb}$ more effectively elevated the production of IFN- $\gamma$ than EpCAM/CD3 BsAb or MUC-1/CD3 BsAb alone (Fig. 7A), suggesting that the proinflammatory effects of BsAbs be associated with the expression of specific antibodies on the surface of primary tumor cells. In addition, combination of EpCAM/CD3 BsAb and MUC-1/CD3 BsAb more effectively elevated the production of IL-6 than EpCAM/CD3 BsAb alone

(A)

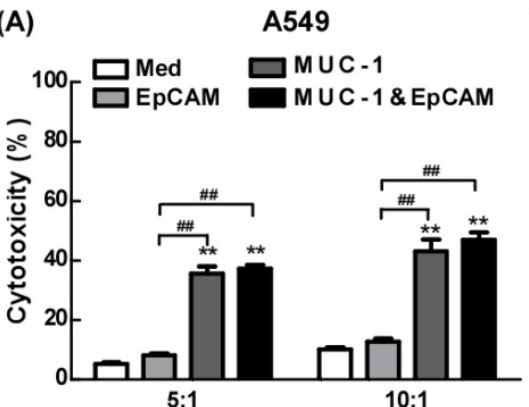

(B)

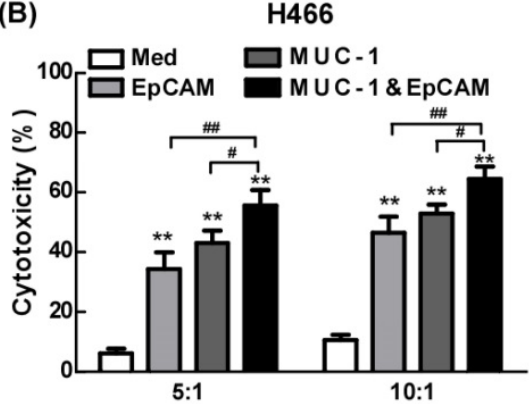

(C)

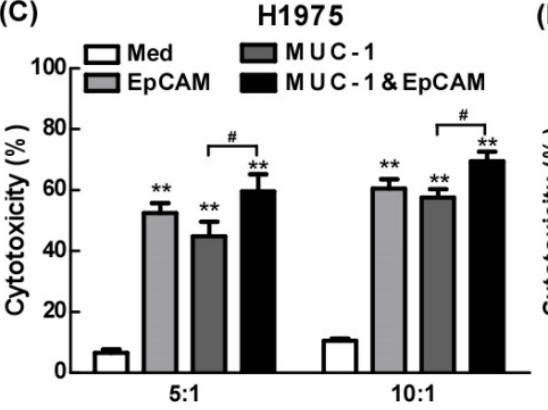

(D)

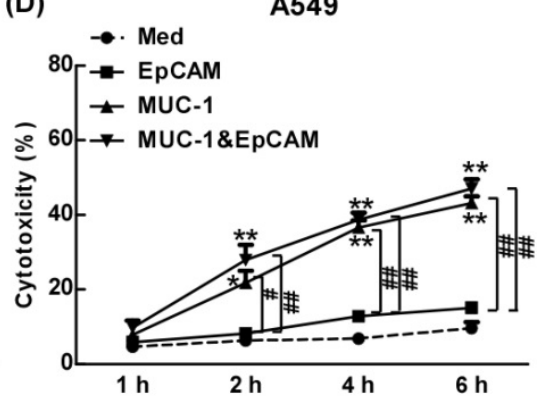

(E)

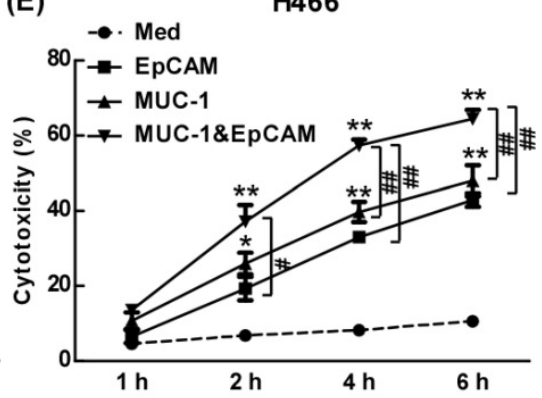

(F)

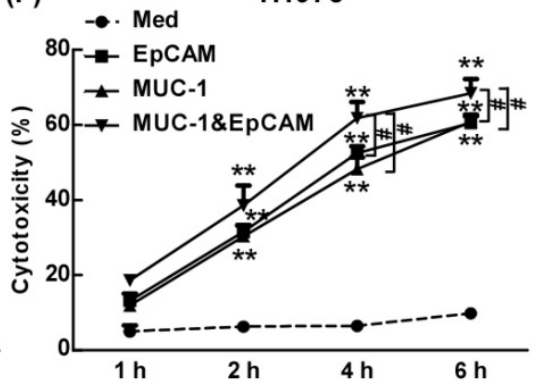

Figure 3. The effect of EPCAM/CD3 BsAb complexed with MUC-1/CD3 BsAb on cytotoxic T-lymphocyte (CTL) response in lung tumor cell lines. In vitro CTL responses were analyzed using non-radioactive cytotoxicity assay in the different BsAbs treated T cells and A549 (A), H466 (B) and H1975 (C) at various effector/target (E: T) ratios. In vitro CTL responses were analyzed using non-radioactive cytotoxicity assay in the different BsAbs treated T cells and A549 (D), H466 (E) and H1975 (F) at different times. Bars shown are mean $\pm S E(n=3-4)$, and differences between medium and other groups are determined using one-way ANOVA analysis. *: $p<0.05 ; * *: p<0.01$. Differences between two different groups are statistically different, $\#: p<0.05$ \#\#: $\mathrm{p}<0.01$
(Fig. 7B). The result was consistent with which observed in cancer cell lines. Overall, combination of EpCAM/CD3 BsAb and MUC-1/CD3 BsAb have induced cytokine production in primary tumor cells.

\section{Combination of EpCAM/CD3 BsAb and MUC-1/CD3 BsAb potently suppressed tumor growth in vivo}

To appraise the effect of combination of EpCAM/CD3 BsAb and MUC-1/CD3 BsAb in vivo, tumor bearing mice (H1975) were randomly divided into four groups and received treatment as outlined in BsAb and MUC-1/CD3 BsAb significantly reduced tumor volume (Fig. 8B). However, combination of EpCAM/CD3 BsAb and MUC-1/CD3 BsAb were more potent than EpCAM/CD3 BsAb or MUC-1/CD3 $\mathrm{BsAb}$ alone to suppress tumor growth in mice (Fig. 8B). Noteworthy, activated $\mathrm{T}$ cells by anti-CD3 and anti-CD28 could not induce effective antitumor effect. In addition, we also evaluated the anti-tumor effects of combination of EpCAM/CD3 BsAb and MUC-1/CD3 BsAb on A549 (Fig. 8C) and H466 (Fig. 8D) tumor bearing mice, respectively. The results showed that the effect of EpCAM/CD3 BsAb could not inhibit tumor growth, which was due to the low expression of EpCAM in A549 (Fig. 8C). However, MUC-1/CD3 BsAb alone or combination of EpCAM/CD3 BsAb and MUC-1/CD3 BsAb significantly reduced tumor volume (Fig. 8C). In addition, the therapeutic effect of different BsAbs in H466 tumor bearing mice was similar with H1975 tumor bearing mice (Fig. 8D).

As is known, tumor-draining lymph nodes (TDLNs) were critical site for generating tumor-specific immune responses [21]. The regulatory $\mathrm{T}$ (Treg) cells in TDLNs were known to suppress immune responses towards tumors, playing a key role in tumor progression and tumor immune escape [22-23]. In addition, effectively activated $\mathrm{CD} 88^{+} \mathrm{T}$ cells have resulted in prolonged tumor regression and improved overall response rates in lung cancers [24]. Previous studies have shown that increasing $\mathrm{T}$ cells in 
TDLNs was crucial for eliciting effective anti-tumor immune responses and tumor eradication [25]. We
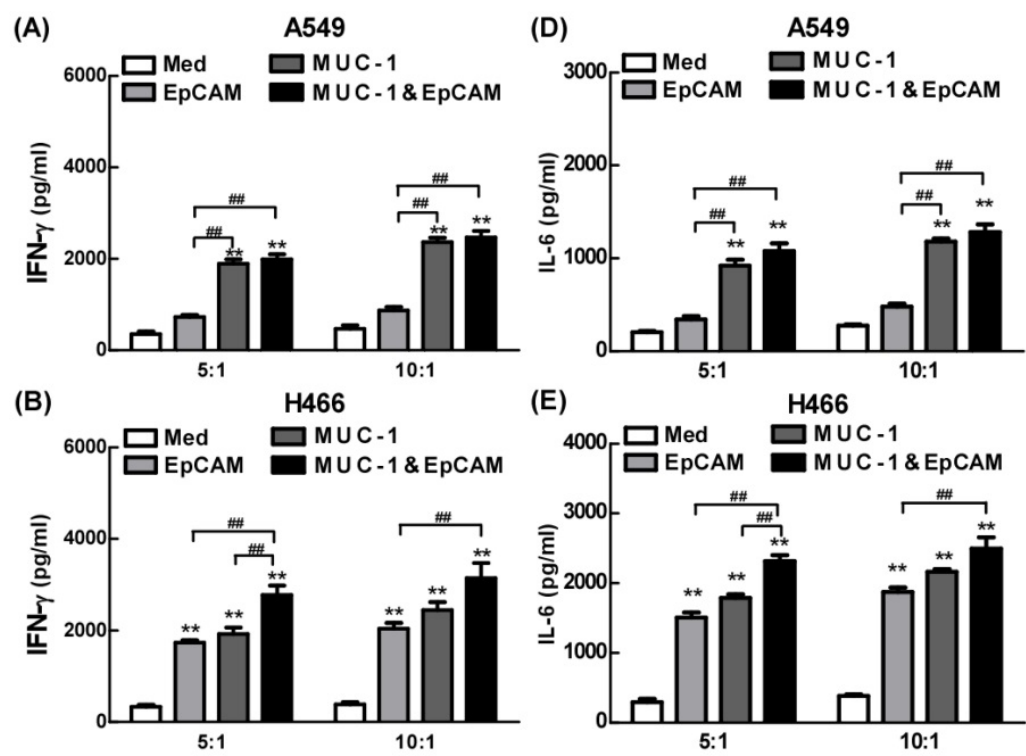

(E)

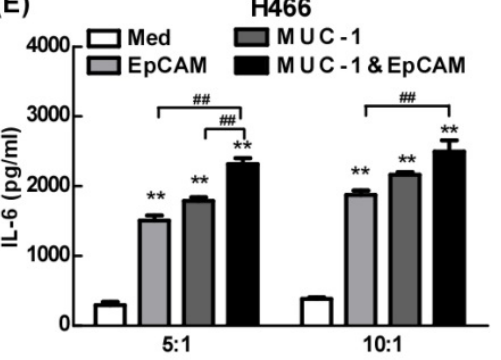

(C)

(F)
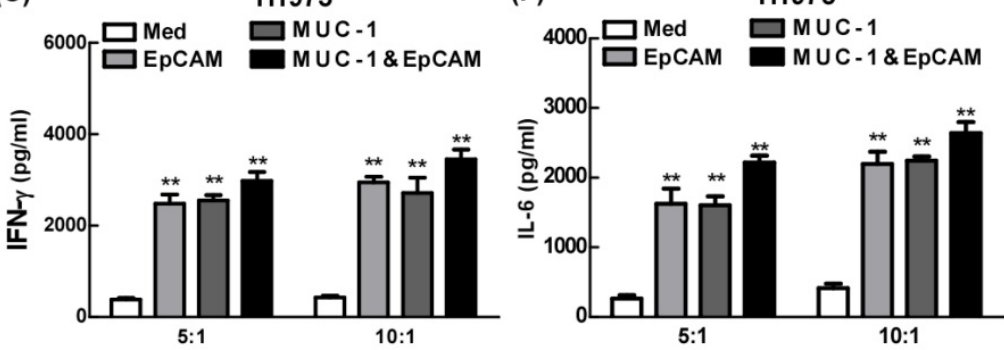

Figure 4. The proinflammatory effects of EpCAM/CD3 BsAb complexed with MUC-1/CD3 BsAb in lung tumor cell lines. The productions of IFN-Y (A-C) and IL-6 (D-F) in culture supernatants were measured using ELISA. Bars shown are mean $\pm S E(n=3-4)$, and differences between medium and other groups are analyzed using one-way ANOVA analysis. **: $p<0.01$. Differences between two different groups are statistically different, \#\#: $\mathrm{p}<0.01$.

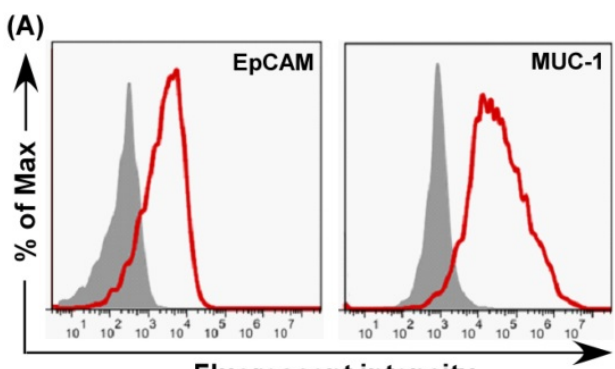

Fluorescent intensity

(B) Primary tumor

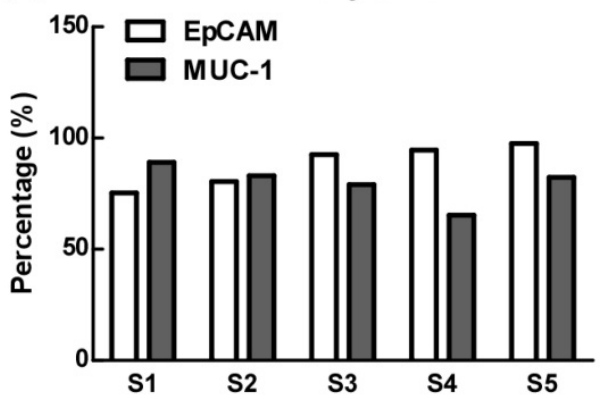

Figure 5. The expression of EpCAM and MUC-1 in primary tumor. Experiments were repeated five times in triplicate each time $(n=5)$ therefore investigated the effect of different $\mathrm{BsAb}$ on $\mathrm{T}$ cell population in TDLNs. However, the immunodeficient mice could not be used for further detection of $\mathrm{T}$ cell population, so we applied the H1975 tumor bearing BALB/c mice model. The activated T cells (aT cells) were harvested after cultured with anti-mouse CD3 antibody and anti-mouse CD28 antibody followed by IL-2 for $72 \mathrm{~h}$. And then the aT cells were treated with or without EpCAM/CD3 BsAb or MUC-1/CD3 BsAb followed by IL-2 for $72 \mathrm{~h}$ to acquire different BsAb treated aT cells (Supplementary methods). Our results showed that as compared with PBS treated H1975 tumor bearing BALB/c mice, the combination of EpCAM/CD3 $\mathrm{BsAb}$ and MUC-1/CD3 BsAb reduced the number of Tregs in TDLNs by $15-20 \%$ (Fig. S1B) and significantly increased the number of $\mathrm{CD}^{+} \mathrm{T}$ cells (Fig. S1C). These results indicated that the function of $\mathrm{T}$ cells in TDLNs has been restored after treatment with combination of EpCAM/CD3 BsAb and MUC-1/CD3 BsAb. Although the model of subcutaneous transplantation of H1975 tumor bearing BALB/c mice might not exactly reproduce the immunosuppressive effect of immune microenvironment in lung cancer patients, our results demonstrated that combination of EpCAM/CD3 BsAb and MUC-1/CD3 $\mathrm{BsAb}$ could trigger a strong immunotherapeutic and improve immune microenvironment in tumor-bearing mice, which might play an important role in anti-tumor therapy.

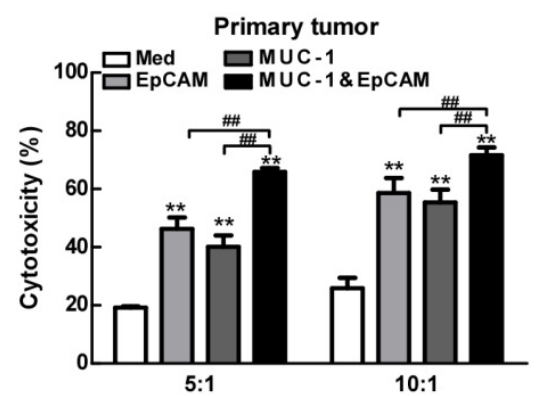

Figure 6. The effect of EpCAM/CD3 BsAb complexed with MUC-1/CD3 BsAb on cytotoxic T-lymphocyte (CTL) response in primary tumor. In vitro CTL responses were analyzed using non-radioactive cytotoxicity assay in A549 (A), H466 (B) and H1975 (C). Bars shown are mean \pm SE $(n=3-4)$, and differences between medium and other groups are determined using one-way ANOVA analysis. ${ }^{* *}: \mathrm{p}<0.01$. Differences between two different groups are statistically different, \#: $\mathrm{p}<0.01$. 

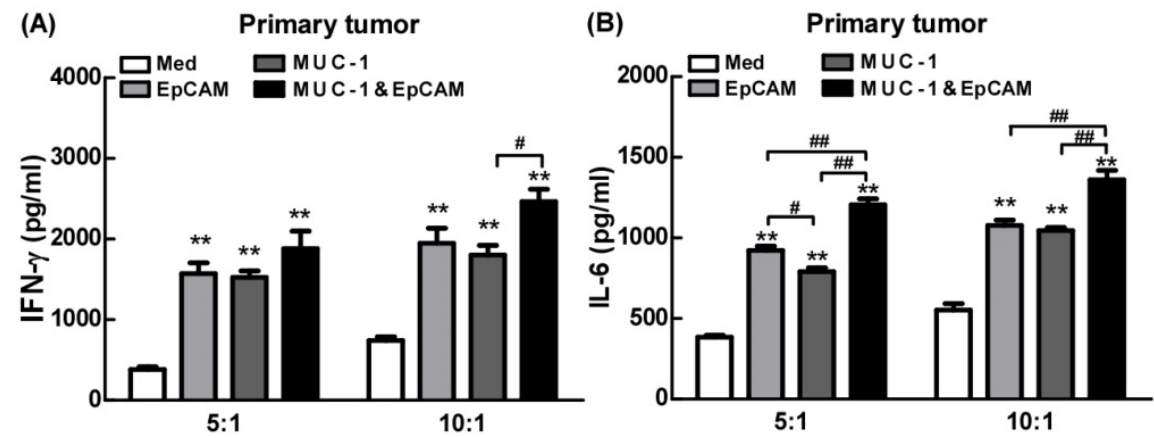

Figure 7. Proinflammatory effects of EpCAM/CD3 BsAb complexed with MUC-1/CD3 BsAb in primary tumor. The productions of IFN-y (A) and IL-6 (B) in culture supernatants were measured using ELISA. Bars shown are mean $\pm S E(n=5)$, and differences between medium and other groups are analyzed using one-way ANOVA analysis. $* *: p<0.01$. Differences between two different groups are statistically different, \#: $p<0.05 ; \# \#$ : $p<0.01$.
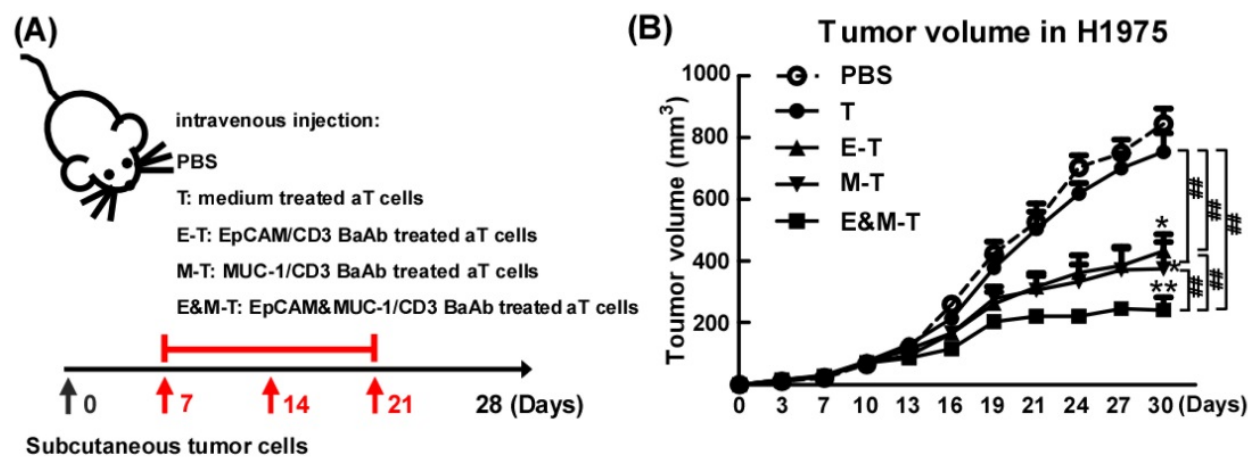

(C)

(D)
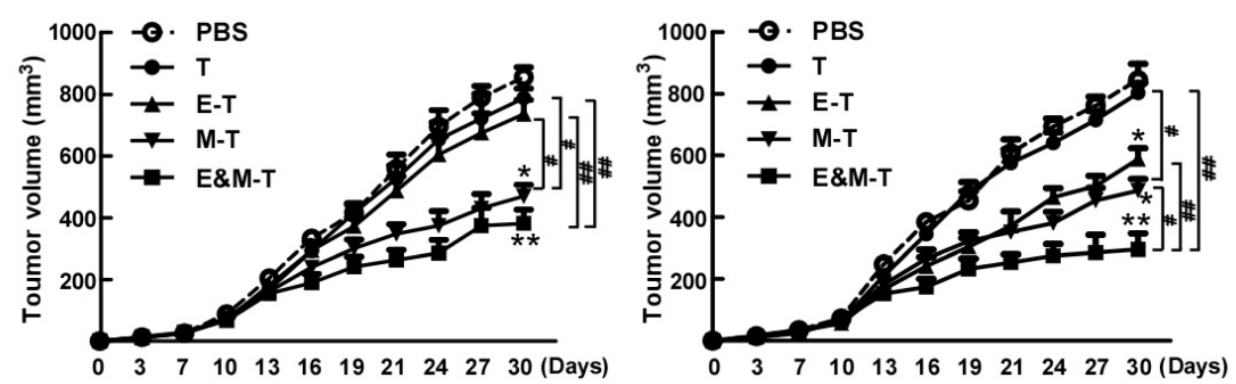

Figure 8. The EPCAM/CD3 BsAb complexed with MUC-1/CD3 BsAb contributes to anti-tumor effects in vivo. Tumor-bearing mice were treated with PBS, medium treated aT cells, EpCAM/CD3 BsAb treated aT cells, MUC-1/CD3 BsAb treated aT cells or EpCAM/CD3 BsAb \& MUC-1/CD3 BsAb treated aT cells. The treatment plan was indicated in the graphs (A). The $\mathrm{H} 1975$ tumor volumes $(B)$, the $A 549$ tumor volumes $(C)$ and the $\mathrm{H} 466$ tumor volumes $(D)$ were monitored every 3 days. Bars shown are mean \pm SE ( $=5$ ), and differences between medium and other groups are determined using one-way ANOVA analysis. *: $p<0.05 ; * *$ : $<<0.01$. Differences between two different groups are statistically different, \#: $\mathrm{p}<0.05 ; \#$; $\mathrm{p}<0.01$.

\section{Conclusions}

Herein, we described a novel immunotherapy strategy by directly targeting MUC-1 and EpCAM in tumor cells through the combination of EpCAM/ CD3 BsAb and MUC-1/CD3 BsAb. The present study showed that combination of EpCAM/CD3 BsAb and MUC-1/CD3 BsAb could dramatically enhance elicited CTL response, as well as promote cytokine production in tumor cell lines and primary tumor cells. More strikingly, combination of EpCAM/CD3 $\mathrm{BsAb}$ and MUC-1/CD3 BsAb effectively improved the immune microenvironment by increasing $\mathrm{CD}^{+} \mathrm{T}$ cells but decreasing Tregs in TDLNs, thereby led to potent antitumor responses, suggesting its potential advantage for the therapy of targeting EpCAM and
MUC-1 on tumor. Hence, combination therapy with EpCAM/CD3 BsAb and MUC-1/CD3 BsAb was expected to be a promising strategy to enhance the anti-tumor efficacy.

\section{Supplementary Material}

Supplementary material and figure S1.

http://www.medsci.org/v18p3380s1.pdf

Supplementary video $\mathrm{S} 1$.

http://www.medsci.org/v18p3380s2.mp4

\section{Acknowledgements}

This project was supported by the National Natural Science Foundation of China (Grant No. 81602695), the Science and Technology Project of Shenzhen (No. JCYJ20170307095822325, 
JCYJ20170413092711058), the Guangdong Medical Science and Technology Research Foundation (NO. A2020487).

\section{Author contributions}

Ce Wang designed the study, performed the experiments, analyzed the data and wrote the manuscript. Shang Chen and Yingjuan $\mathrm{Wu}$ performed the experiments. Furong $\mathrm{Li}$ and Ce Wang co-designed the study and co-wrote the manuscript.

\section{Competing Interests}

The authors have declared that no competing interest exists.

\section{References}

1. Ahmedin Jemal, Freddie Bray, Melissa M Center, et al. Global cancer ststistics. CA Cancer J Clin. 2011; 61(2):69-90.

2. Maureen $\mathrm{F}$ Zakowski. Analytic inquiry: molecular testing in lung cancer. Cancer Cytopathol. 2017; 125(S6): 470-476.

3. Jean-Charles Soria, Yuichiro Ohe, Johan Vansteenkiste, et al. Osimertinib in untreated EGFR-mutated advanced non-small-cell lung cancer. N Engl J Med. 2018; 378(2): 113-125.

4. Sergey E Sedykh, Victor V Prinz, Valentina N Buneva, et al. Bispecific antibodies: design, therapy, perspectives. Drug Design, Development and Therapy. 2018;12: 195-208.

5. Diane Seimetz Novel monoclonal antibodies for cancer treatment: the trifunctional antibody catumaxomab (removab). J Cancer. 2011; 2: 309-316.

6. Maren Knödler, Justus Körfer, Volker Kunzmann, et al. Randomised phase II trial to investigate catumaxomab (anti-EpCAM $\mathrm{x}$ anti-CD3) for treatment of peritoneal carcinomatosis in patients with gastric cancer. Br J Cancer. 2018; 119(3): 296-302.

7. Klaus Pietzner, Michael Jäger, Alexandra Schoberth, et al. First patient treated with a re-challenge of catumaxomab in recurrent malignant ascites: a case report. Med Oncol. 2012; 29(2): 1391-1396.

8. Hagop Kantarjian, Anthony Stein, Nicola Gökbuget, et al. Blinatumomab versus chemotherapy for advanced acute lymphoblastic leukemia. N Engl J Med. 2017; 376(9): 836-847.

9. Shengnan $\mathrm{Yu}$, Anping Li, Qian Liu, et al. Recent advances of bispecific antibodies in solid tumors. J Hematol Oncol. 2017;10(1): 155.

10. Jung Ho Kim, Jeong Mo Bae, Young Seok Song, et al. Clinicopathologic, molecular, and prognostic implications of the loss of EPCAM expression in colorectal carcinoma. Oncotarget. 2016; 7(12): 13372.

11. Anna K Campos, Hilde D Hoving, Stefano Rosati, et al. EpCAM expression in lymph node and bone metastases of prostate carcinoma: a pilot study. Int J Mol Sci. 2016; 17(10): 1650.

12. Meng Dai, Fei Yuan, Cuiqun $\mathrm{Fu}$, et al. Relationship between epithelial cell adhesion molecule (EpCAM) overexpression and gastric cancer patients: a systematic review and metaanalysis. PLoS One. 2017; 12(4): e0175357.

13. Zhidong Liu, Dezhi Zhen, Yunsong Li, et al. Clinical significance of EpCAM in circulating tumor cells in the peripheral blood during adjuvant therapy for postoperative non-small cell lung cancer patients. Chest. 2016; 149(4): 325.

14. Min Gyoung Pak, Dong Hoon Shin, Chang Hun Lee, et al. Significance of EpCAM and TROP2 expression in non-small cell lung cancer. World J Surg Oncol. 2012; 10: 53

15. Joyce A Schroeder, Azzah Al Masri, Melissa C Adriance, et al. MUC1 overexpression results in mamm gland tumorigenesis and prolonged alveol differentiation. Oncogene. 2004; 23 (34): 5739 -5747.

16. A Giatromanolaki, M I Koukourakis, E Sivridis, K O'Byrne, et al. Coexperssion of MUC1 glycoprotein with multiple angiogenic factors in non-small cell lung cancer suggests coactivation of angiogentic and migration the ways. Clin Cancer Res. 2000; 6(5): 1917-1921.

17. Junya Inata, Noboru Hattori, Akihito Yokoyama, et al. Circulating KL-6/MUC1 mucin carrring sialyl Lewisa oligosaccharide is an independent prognostic factor in patients with lung adenocarcinoma. Int J Cancer. 2007; 120(12): 2643-2649.

18. Luojun Chen, Min Peng, Na Li, et al. Combined use of EpCAM and FRa enables the high-efficiency capture of circulating tumor cells in non small cell lung cancer. Sci Rep. 2018; 8(1):1188

19. Zichao Luo, Ping Li, Jizhe Deng, et al. Cationic polypeptide micellebased antigen delivery system: a simple and robust adjuvant to improve vaccine efficacy. J Control Release. 2013; 170: 259-67.

20. S A Ghanekar, L E Nomura, M A Suni, et al. Gamma interferon expression in CD8(+) $\mathrm{T}$ cells is a marker for circulating cytotoxic $\mathrm{T}$ lymphocytes that recognize an HLA A2-restricted epitope of human cytomegalovirus phosphoprotein pp 65. Clin Diagn Lab Immunol. 2001; 8(3): 628-31.
21. Stéphanie Hugues, Luc Fetler, Laura Bonifaz, et al. Distinct T cell dynamics in lymph nodes during the induction of tolerance and immunity. Nat Immunol. 2004; 5: 1235-42.

22. Liufu Deng, Haizeng Zhang, Yan Luan, et al. Accumulation of foxp3+ T regulatory cells in draining lymph nodes correlates with disease progression and immune suppression in colorectal cancer patients. Clin Cancer Res 2010; 16: 4105-4112.

23. Shih-Chia Huang, Pei-Chi Wei, Wendy W Hwang-Verslues, et al. TGF- $\beta 1$ secreted by Tregs in lymph nodes promotes breast cancer malignancy via up-regulation of IL-17RB. EMBO Mol Med. 2017; 9(12): 1660-1680.

24. Samit Chatterjee, Wojciech G Lesniak, Matthew Gabrielson, et al. A humanized antibody for imaging immune checkpoint ligand PD-L1 expression in tumors. Oncotarget. 2016; 7:10215-27.

25. Zichao Luo, Ce Wang, Huqiang Yi, et al. Nanovaccine loaded with poly I:C and STAT3 siRNA robustly elicits anti-tumor immune responses through modulating tumor-associated dendritic cells in vivo. Biomaterials. 2015; 38: $50-60$ 